\title{
Le determinanti dei processi di in/outsourcing della funzione commerciale nelle pmi. II caso del settore edile
}

ORONZO TRIO*

\begin{abstract}
Obiettivo del paper: Comprendere ed analizzare le determinanti dei processi di in/outsourcing di un campione di piccole imprese operanti nel settore delle costruzioni $e$, specificamente, nel mercato residenziale.

Metodologia: Multiple case study approach con interviste in profondità.

Risultati: I risultati raggiunti hanno consentito di individuare un prevalente orientamento nel campione esaminato verso l'internalizzazione della funzione commerciale al fine di gestire in modo diretto il rapporto con il cliente e per guidarlo nella scelta del prodotto abitativo più confacente alle sue necessità.

Limiti della ricerca: La ricerca ha interessato esclusivamente un campione di piccole imprese ponendo così la necessità di estenderlo alle grandi per verificare se vi siano differenze nell'approccio adottato.

Implicazioni pratiche: Il lavoro fornisce degli elementi interessanti tanto per il management delle imprese di costruzioni che per le agenzie immobiliari, in quanto pone in risalto le peculiari problematiche inerenti la funzione commerciale e le relazioni con la clientela finale.

Originalità del lavoro: Il presente lavoro fornisce un primo contributo volto a colmare il gap riscontrato negli studi di management sul tema dell'outsourcing e dei rapporti industriadistribuzione nel settore delle costruzioni.
\end{abstract}

Parole chiave: outsourcing; industria-distribuzione; settore edile; prodotto abitativo

Purpose of the paper: The aim of the paper is to understand and to analyse the determinants of the in/outsourcing processes in a sample of small and medium enterprises in the residential markets.

Methodology: Multiple case study approach by in-depth interviews.

Findings: The findings reveal in the sample a prevalent attitude to insource the selling function in order to have a direct relationship with the customers and to suggest them the most suitable residential product.

Research limits: The research involved only small and medium enterprises so it needs to be extended to large firms in order to verify if any differences exist in the approach adopted.

Professore Aggregato di Marketing Territoriale - Università del Salento

e-mail: oronzo.trio@unisalento.it 
Practical implications: The paper presents some interesting elements both for the building enterprises management and for the real estate agents because it highlights the specific problems of selling activities and the customer relationship.

Originality of the paper: This work provides a first contribution to bridge the gap found in the management studies on the topic of outsourcing processes and on the trade-industry relationships in the construction industry.

Key words: outsourcing; trade-industry relationship; construction sector; residential product

\section{Introduzione}

Il settore delle costruzioni è stato scarsamente indagato negli studi di marketing; se si escludono alcuni pur autorevoli contributi, (Sabbadin, 2007; Castaldo e Sabbadin, 2009; Baldini, 2010), manca un corpus teorico di riferimento che aiuti a comprendere le dinamiche del comparto. Sono peraltro del tutto assenti lavori riguardanti specificamente lo studio delle politiche distributive delle imprese di costruzioni, in particolare nel comparto dell'edilizia residenziale, e più in generale $\mathrm{i}$ rapporti industria-distribuzione che, al contrario, hanno ricevuto ampia attenzione da parte degli studiosi con riferimento ad altri mercati.

Per colmare, seppur parzialmente, questo vuoto, il presente studio, basato su un'indagine qualitativa effettuata attraverso 15 interviste rivolte ad imprenditori del settore, analizza l'orientamento strategico di un campione di piccole imprese operanti nell'ambito dell'edilizia residenziale rispetto alla scelta di in/outsourcing della funzione vendita e delle attività connesse al collocamento sul mercato del prodotto abitativo.

Il lavoro è strutturato nel modo seguente. Il primo paragrafo, utilizzando le chiavi di lettura provenienti dalla teoria dei costi di transazione e dalla agency theory, espone i principali contributi teorici sul tema dell'outsourcing, esponendo potenzialità e criticità insite nelle decisioni adottate dalle imprese. Nel paragrafo successivo si descrivono gli attori operanti nella filiera edile, con specifico riferimento all'impresa di costruzioni ed all'agenzia immobiliare, ponendo in risalto le peculiarità del prodotto immobiliare. Dopo aver riportato nel terzo paragrafo $\mathrm{i}$ criteri metodologici adottati, vengono rappresentati i risultati della ricerca (paragrafi 4 e 5) ed infine le conclusioni e le implicazioni manageriali.

\section{Inquadramento teorico delle opzioni strategiche di in/outsourcing}

I rapporti di filiera tra industria e distribuzione sono stati oggetto di un intenso dibattito nell'ambito della letteratura di marketing che li ha analizzati ed indagati da più prospettive. Tali studi fanno emergere l'inadeguatezza del mercato ad esprimere compiutamente la complessità dei rispettivi sistemi e propongono tre distinte configurazioni, basate rispettivamente su negoziazione, conflitto e cooperazione (Vaccà, 1963; Varaldo, 1971; Spranzi, 1972; Lugli, 1976; Varaldo e Dalli, 1989; 
Pastore, 1996; 2000; Varaldo e Dalli, 2003; Golinelli, 2004; Castaldo, 2005; Longo, 2006; Gandolfo e Sbrana, 2007; Floreddu e Cabiddu, 2013).

Secondo tali contributi, a determinare la struttura delle relazioni è il grado di controllo che gli attori riescono ad esercitare sul canale: si ipotizzano così rapporti fondati su basi collaborative, in presenza di uno squilibrio in favore di uno dei partner che stabilisce le regole di funzionamento del canale, e relazioni conflittuali quando la ripartizione del potere è equilibrata ed i due soggetti cercano di imporsi reciprocamente politiche e condizioni del rapporto (Massaroni e Ricotta, 2009; Rullani, 2010) ${ }^{1}$.

In generale i fattori di conflitto possono riguardare:

(a) la politica delle scorte;

(b) la politica di marca;

(c) la politica del prodotto e degli assortimenti;

(d) la politica di prezzo;

(e) la gestione dei flussi informativi.

Per attenuare il grado di conflittualità può essere molto importante il ruolo della fiducia che rappresenta il "livello di certezza che la controparte adotterà in futuro un comportamento in linea con le attese o con ciò che aveva promesso"; si tratta di un elemento centrale nella relazione, un costrutto concettuale che ne coglie la natura dinamica e contingente, ne incorpora i significati essenziali (Castaldo, 2005, p. 206), e ne costituisce il presupposto per comportamenti collaborativi e per la riduzione dell'incertezza ambientale (Bagozzi, 1974; 1975; Dwyer et al., 1987; Nevins e Money, 2008; Maizza et al., 2012; Fait, 2012).

Tuttavia quando la fiducia si riferisce al rapporto con il mercato, sostanziandosi in risorse esterne accumulate nel tempo dall'impresa, può condurre a uno sbilanciamento del potere: la capacità di una delle parti (produttore o distributore) di raggiungere un elevato livello di fiducia da parte dei clienti (effettivi o potenziali) consente in genere di condizionare i comportamenti dell'altra parte.

Questo potere mette chi lo detiene nella condizione di porsi quale leader in grado di conseguire un comportamento coordinato dei soggetti, per cui un'impresa industriale con un marchio forte sarà in grado di dominare un distributore che non ha saputo sviluppare una consistente fedeltà all'insegna, così come un distributore con un consistente potere di mercato saprà influenzare i comportamenti di un fornitore secondario, realizzando un coordinamento delle azioni di marketing (Castaldo, 1994).

Dunque la fiducia è la variabile chiave di mediazione tra i membri del canale e, proiettata in un contesto internazionale, in mercati in cui interagiscono soggetti portatori di esigenze differenti, rappresenta la soluzione al problema della diversità di cultura e di linguaggi tipica di chi opera in mercati diversi dal proprio (Morgan e

1 Interessante è anche l'analisi condotta da Guido (2000), il quale ricostruisce la dinamica dei rapporti, distinguendo il caso della cooperazione da quello della defezione del produttore, del distributore o di entrambi, a seconda delle diverse finalità perseguite e delle differenti condizioni di contesto in cui si trovano ad operare. 
Hunt, 1994; Geyskens et al., 1996; Sullivan e Peterson, 1982; Jap e Anderson, 2003; Kiessling e Harvey, 2004; Grewal et al., 1999).

La considerazione degli elementi appena esposti introduce un ulteriore elemento di riflessione che riguarda i processi di in/outsourcing, tema anche questo ampiamente discusso e dibattuto dalla letteratura, in particolare nell'ambito della teoria dei costi di transazione e della agency theory (Coase, 1937; Williamson, 1978; Quinn e Hilmer, 1994; Hinterhuber e Stuhec, 1996; Mullin, 1996; Elmuti et al., 1998; Baden-Fuller et al., 2000; Ricciardi, 2001; Gregori, 2001; Ventricelli, 2004; Le Bon e Hughes, 2009; Pellicelli, 2009; Pastore e Ricciardi, 2010).

La teoria dei costi di transazione tende a definire i confini dell'impresa sulla base delle migliori performance conseguibili esternalizzando determinate attività aziendali o, al contrario, svolgendole internamente ${ }^{2}$. La agency theory postula invece l'esistenza di una relazione tra due parti di cui una, detta principale, affida ad un'altra (detta agente), lo svolgimento per suo conto di specifiche funzioni. Il focus è posto sulla determinazione del contratto più efficiente per governare una particolare relazione, date le caratteristiche delle parti e l'incertezza ambientale che rende più elevati i costi delle informazioni e più complesso il monitoraggio delle attività dell'agente da parte del principale.

Queste caratteristiche emergono ogniqualvolta, a causa delle asimmetrie informative, il principale non sia in grado di monitorare facilmente l'azione dell'agente, non sia in possesso di informazioni adeguate sulle sue prestazioni e non riesca per tale ragione a quantificare in che misura gli sforzi di quest'ultimo abbiano contribuito al raggiungimento dell'obiettivo ${ }^{3}$.

La teoria dell'agenzia presuppone che gli individui agiscano prevalentemente in difesa del proprio interesse per massimizzare l' utilità personale, e che principale ed agente siano caratterizzati da una differente propensione al rischio.

I rapporti industria/distribuzione e le conseguenti opzioni di in/outsourcing delle attività commerciali per le imprese industriali, hanno ricevuto dalla strumentazione teorica della transactional analysis cost e della agency theory, un significativo contributo.

Le posizioni emerse in letteratura su quale debba essere l'orientamento strategico da preferire non sono univoche. Alcuni autori sostengono la tesi della vantaggiosità del ricorso esclusivo alla forza vendita esterna (Dishman, 1996; Weinrauch et al., 1997; Jackson et al., 1999; McQuiston, 2001; Gulati e Bristow, 2005; Ross et al., 2005), in quanto l'outsourcing consentirebbe in questi casi di: (i) liberare risorse da

2 Di particolare significato è stato inoltre il contributo di Prahalad e Hamel (1990) con la loro concettualizzazione delle "core competencies" e delle collegate "distintive capabilities" utili a separare le funzioni strategiche da quelle non strategiche.

3 La divergenza di preferenze tra principale e agente, unita alle informazioni private delle quali quest'ultimo dispone, causa inoltre una distorsione delle informazioni generando il fenomeno denominato moral hazard, o rischio morale, per cui un agente è motivato dalla natura stessa del sistema di controllo a comunicare in maniera distorta al principale le informazioni rilevanti (Anthony et al., 2006). 
destinare ad altri scopi, (ii) condividere i rischi, (iii) trarre benefici dalle migliori performance realizzabili da imprese specializzate, (iv) eliminare inefficienze interne, (v) utilizzare risorse non disponibili internamente, (vi) ridurre i costi di controllo, (vii) generare liquidità dalla vendita delle funzioni esternalizzate, (viii) rendere disponibile il capitale così liberato per concentrarlo su altre attività core (Elmuti et al., 1998).

Secondo i sostenitori di questa posizione l'esternalizzazione della forza vendita offrirebbe il vantaggio di affidare a soggetti specializzati il compito di mediare il dialogo tra l'impresa ed i clienti, di approfondire la conoscenza dei mercati di riferimento, richiedendo al contempo di vigilare sulla qualità percepita dal cliente, sul suo pieno e totale coinvolgimento durante il processo d'acquisto, ma anche sui processi operativi interni delle imprese alle quali vengono demandate le attività commerciali, essendo indispensabile esercitare un controllo ed un monitoraggio costanti (Rajagopal, 2008; Li, 2012).

Altri autori propongono l'adozione di un sistema integrato che faccia coesistere i canali distributivi esterni ed un proprio canale interno; quest'ultima soluzione, pur incrementando i costi di transazione nel breve termine, consentirebbe comunque una riduzione dei costi di opportunità nel lungo periodo (Kabadayi, 2011).

Una posizione che invece propende per l'insourcing è quella di chi evidenzia la necessità, in presenza di prodotti complessi per i quali esiste un gap di conoscenza da parte dell'acquirente, di gestire internamente la forza vendita, essendo quest'ultima determinante proprio per colmare il gap conoscitivo e per dare, attraverso la maggiore qualificazione e competenza, risposte esaurienti e soddisfacenti al cliente (Rapp, 2009).

Seguendo questa linea di pensiero, Kotable et al. (2012) affermano che la funzione vendita non debba essere esternalizzata per i rischi che l'impresa correrebbe di subire un ridimensionamento delle barriere competitive legate alla gestione diretta dei rapporti con i clienti. A simili conclusioni giungono anche Thelen et al. (2011) e Kalaignanam e Varadarajan (2012), le cui ricerche evidenziano come l'outsourcing delle attività di marketing generi un impatto negativo sulla qualità percepita dal cliente e sulla sua soddisfazione e fedeltà. Secondo questi autori dunque vi sarebbe una relazione negativa tra outsourcing del CRM e performance conseguibili nella relazione con il cliente.

Da ultimo, appare particolarmente interessante quanto sostenuto da Freytag et al. (2012), secondo i quali l'insourcing può essere una delle possibili soluzioni alternative all'outsourcing quando quest'ultima scelta, già compiuta in passato, si sia col tempo rivelata sbagliata. Questa ipotesi determina quattro distinti percorsi alternativi che l'impresa potrebbe seguire: (i) mantenere lo stesso partner, modificando i contenuti del contratto; (ii) chiudere il contratto ed affidare le stesse attività ad un altro partner; (iii) re-internalizzare le attività; (iv) creare una nuova unità di business (es. spin-off). 


\section{La filiera edile ed il prodotto abitativo}

Nel mercato residenziale la decisione in merito alla esternalizzazione o, al contrario, alla internalizzazione della forza vendita, deve essere assunta considerando le specificità del prodotto scambiato e la natura e le attività dei soggetti che partecipano al processo.

Il prodotto immobiliare è tipicamente un prodotto complesso. Presenta un elevato rischio finanziario, richiede un impegno temporale ed un coinvolgimento nella scelta d'acquisto ben più elevati rispetto ad altre tipologie; inoltre per il suo acquisto vi sarebbe, secondo alcuni, una particolare propensione a "trattare" con il personale di vendita interno dell'impresa costruttrice e venditrice (Jackson et al., 1999; Bitner, 1990).

La casa ha subìto nel corso dell'ultimo secolo dei profondi mutamenti nelle tecniche di costruzione e nelle scelte architettoniche, frutto della trasformazione delle sue funzioni e della evoluzione da semplice struttura fisica a prodotto economico, bene sociale e pacchetto di servizi.

Essa incorpora una pluralità di significati materiali, emotivi e sentimentali: la sua complessità (Tidd et al., 1999) è cresciuta di pari passo con l'emergere di nuovi bisogni come quelli legati al contenimento dei consumi energetici ("casa a impatto zero") $)^{4}$, all'isolamento acustico ed a tutto il complesso di fattori che riguardano l'utilizzo degli spazi interni (Finizio, 2011; Morello, 2011) ed i differenti stili abitativi di chi la vive (Abis, 2009).

La casa è però anche altro. È un prodotto, cioè l'output del processo produttivo di un'impresa che combina materiali, capitale, suolo, lavoro, abilità imprenditoriale,

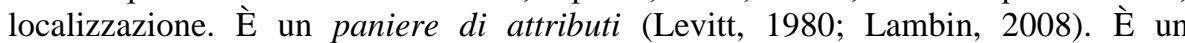
complesso di utilità (funzionali, finanziarie, sociali e simboliche).

La considerazione di tutti questi fattori fa comprendere la natura particolarmente complessa del processo d'acquisto e le conseguenti difficoltà che un'impresa di costruzioni deve affrontare nella relazione con il cliente finale, in special modo quando la trattativa avvenga "su carta" (Gameson, 1991; Loe, 2000).

La conoscenza del cliente implica infatti che si studi come lui vede il mondo, come e cosa pensa, come decide, su quali criteri assume le sue scelte, come agisce all'interno del proprio ambito di riferimento. Cliente ed impresa infatti guardano alla stessa cosa ma vedono cose differenti, usano le stesse parole ma attribuiscono significati diversi, quando il cliente osserva il progetto di un edificio lo guarda principalmente con riferimento al suo sistema di valori e non ai dettagli della costruzione.

La natura del prodotto e l'impatto del suo acquisto sul piano finanziario e su quello funzionale inoltre fanno si che i soggetti coinvolti nella scelta, che gli studi di

4 Ne è conseguito un nuovo concetto di casa che rispetta prefissati limiti di fabbisogno di energia primaria per la climatizzazione invernale, e che si adegua ai valori imposti per la trasmittanza termica (flusso di calore che passa attraverso una parete), sia per le pareti che per le coperture (Trio, 2008; 2010a; 2010b). 
marketing tradizionalmente classificano in influenzatori, iniziatori, decisori, compratori ed utilizzatori, si concentrino prevalentemente nell'ambito dello stesso nucleo familiare, esercitando, rispetto alla decisione finale, un'influenza ben più efficace di qualunque altro stimolo proveniente dall'esterno, come la pubblicità, le attività promozionali nonché quelle poste in essere da costruttori, immobiliaristi ed agenzie immobiliari (Brassington e Pettitt, 2000) ${ }^{5}$.

La filiera edile si presenta come una filiera lunga, complessa ed articolata. $\mathrm{Ne}$ fanno parte settori e comparti interconnessi corrispondenti a differenti ambiti produttivi ed a distinti segmenti di mercato.

Le principali categorie possono essere distinte in primo luogo tra:

- proprietari-utilizzatori, che gestiscono immobili di proprietà strumentali al proprio business;

- proprietari-investitori, per i quali la gestione del patrimonio immobiliare rappresenta il core-business che hanno obiettivi di tipo strettamente finanziario od operativo;

- fornitori di servizi di asset management, che supportano i proprietari-investitori nella gestione finanziaria del patrimonio;

- fornitori di servizi immobiliari, che si occupano prevalentemente della compravendita degli immobili (le agenzie);

- costruttori;

- studi di progettazione e general contractor, che forniscono servizi di progettazione, ingegneria e gestione delle attività di costruzione e di cantiere;

- fornitori di servizi di property e facility management per conto dei proprietari, quali servizi amministrativi su beni immobili, gestione dei fornitori per i servizi di manutenzione ecc. (Politecnico di Milano, 2009).

L'impresa di costruzioni si colloca all'interno delle cosiddette imprese progetto, vale a dire di quella tipologia di imprese che presentano (i) unicità e non riproducibilità del prodotto, (ii) intermittenza del processo, (iii) transitorietà del sistema organizzativo, (iv) natura non routinaria delle transazioni, (v) flessibilità delle strutture (Genco, 2006; Trio, 2010).

È un'impresa "service enhanced", non offre cioè semplici prodotti manifatturieri, ma soluzioni complete a specifiche "domande" del cliente, mediante sistemi che integrano elementi fisici relativi al prodotto ed elementi immateriali relativi al servizio, tra loro complementari.

Il suo mercato è segmentabile in base all'oggetto dell'intervento (edilizia o genio civile, costruzione ex novo o riuso), ed al soggetto che esprime la domanda ${ }^{6}$.

5 La famiglia, per la sua ristretta dimensione, consente interazioni face-to-face dense di contenuti, attiva un "word-of-mouth" personale con un impatto ben più significativo di quello di altri influenzatori (Brown e Reingen, 1987; Engel et al, 1995), riduce il rischio percepito nell'acquisto, induce i membri della famiglia a porre in essere un processo decisionale congiunto (Antonides e Van Raaij, 1988; Wagner et al, 1984).

6 In particolare, a seconda della natura pubblica o privata sono individuabili: (Albino et al., 2000): committenti pubblici (amministrazioni centrali, enti locali, concessionarie 
Il progetto rappresenta l'unità di analisi elementare, qualificandosi in due modi distinti ma collegati: il progetto come design (progetto concepito) ed il progetto come realizzazione ${ }^{7}$.

Le agenzie immobiliari costituiscono l'anello di collegamento con il mercato, trattandosi di intermediari commerciali che intervengono per coprire il vuoto informativo del mercato residenziale (Milgrom e Roberts, 1994; Grandori, 1999; Boyd e Chinyio, 2006).

Costituiscono una particolare categoria di intermediari commerciali la cui originalità risiede sia nella natura del prodotto che concorrono a far scambiare sia nella loro specifica "identità".

Agiscono su impulso di un cliente che conferisce un mandato ad operare per suo conto con l'obiettivo di individuare sul mercato persone, imprese od organizzazioni che abbiano l'interesse a concludere una determinata transazione in qualità di acquirenti o venditori di un immobile o, nel caso di locazione, di locatari o locatori.

Essi si configurano quindi come distributori di soluzioni immobiliari il cui livello di competitività è strettamente collegato alla ampiezza dell'assortimento, vale a dire alla numerosità ed alla varietà delle soluzioni immobiliari messe a disposizione della clientela.

Le loro funzioni sono quelle tipiche degli intermediari commerciali in generale: in primo luogo esercitano una funzione di contatto con il mercato, quindi con chi è interessato ad avere $\mathrm{o}$ a cedere la disponibilità dell'immobile.

In secondo luogo si occupano della funzione informativa, trasferendo alla parte con la quale entrano in contatto un flusso di notizie di carattere tecnico, funzionale, finanziario, economico ecc., utili al processo decisionale della controparte ed alla verifica circa la rispondenza dell'offerta alle esigenze ed ai bisogni che l'immobile è in grado di soddisfare.

In terzo luogo svolgono una funzione di frazionamento dell'offerta delle imprese immobiliari, quindi di scomposizione del complessivo "pacchetto" nei vari lotti e nelle diverse unità rispondenti ciascuna alle esigenze di specifici segmenti o nicchie.

In quarto luogo si occupano delle attività promozionali mediante il ricorso ad una strumentazione alquanto differenziata ed eterogenea (cartellonistica, brochure, siti dedicati). Infine ricoprono un ruolo essenziale per tutte quelle attività di tipo amministrativo e legale (quali a titolo di esempio la predisposizione dei preliminari di vendita, la ricezione di pagamenti in acconto, le ricerche catastali, l'assistenza in fase di redazione del rogito ecc.).

pubbliche) e committenti privati (famiglie, imprese immobiliari, condomini, artigiani e piccole imprese, grandi imprese).

7 La separazione spazio-temporale tra il momento della concezione dell'opera e quello della sua realizzazione aggiunge specifici elementi di complessità per il marketing, in quanto la strategia commerciale dovrà incorporare le difficoltà connesse con l'oggetto della vendita, o della trattativa più in generale: la sua prima concettualizzazione potrà avere quindi, modifiche anche notevoli nel corso della realizzazione dell'opera (Norsa, 2006). 
Il rapporto con il cliente finale è di vitale importanza per l'impresa di costruzioni quanto lo è per lo stesso cliente la scelta della casa che meglio risponda ai suoi bisogni ed alle proprie esigenze di vita.

L'intervento dell'agente immobiliare condiziona, in negativo o in positivo per il produttore, questa scelta orientandola verso determinate soluzioni abitative offerte dall'impresa o, al contrario, su altre.

L'agente può adottare comportamenti opportunistici, agire sul prezzo di vendita, fare leva su variabili psicologiche ed emotive dell'acquirente, allontanandolo dall'interesse del principale.

È per questo che il mantenimento ed il controllo della relazione costituisce una variabile chiave per l'impresa di costruzioni che dovrà attentamente valutare quale sia il percorso più consono riguardo alla gestione della forza vendita.

\section{Aspetti metodologici}

La ricerca è basata su una indagine di tipo qualitativo realizzata attraverso 15 interviste semi-strutturate a manager, direttori tecnici o commerciali di aziende di costruzioni operanti nel territorio pugliese.

L'adozione dell'approccio qualitativo è stato ritenuto il più consono agli obiettivi di uno studio in profondità e ad una comprensione più ampia del fenomeno, considerata la finalità esplorativa ed induttiva della ricerca. Si è fatto ricorso ad un multiple case study approach (Malhotra, 1993; Johnston et al., 1999; Yin, 1984; Gummesson, 2000), soprattutto per la particolare natura dell'unità di analisi, quali sono le organizzazioni e le reciproche relazioni tra di esse (nel nostro caso le imprese edili e le agenzie immobiliari).

Numerosi contributi teorizzano la superiorità, in tali casi, dei metodi di ricerca qualitativi. Un case study di una singola entità, o di un ridotto numero, dà infatti accesso ad una grande quantità di informazioni, prevalentemente qualitative, ed offre indicazioni sulla natura dei fenomeni ben più ampie di quanto non potrebbe ottenersi con metodi quantitativi (Dubois e Gibbert, 2010; Easton, 2010; Piekkari et al., 2010; Dubois a Gadde, 2013).

Lo stesso Yin (1984) considera il multiple case study un metodo più efficace rispetto al singolo case study poiché i risultati che ne derivano sono "più "robusti". Una posizione questa ripresa anche da Eisenhardt $(1989 ; 1991)$ il quale sottolinea come "un numero compreso tra 4 e 10 casi generalmente vada bene" senza necessità di ulteriori argomentazioni. Scegliere dunque più casi nella ricerca consente di sviluppare una teoria più elaborata, di disegnare un quadro teorico più completo e di elaborare una teoria "più fondata, più accurata e più generalizzabile" (Eisenhardt $\mathrm{e}$ Graebner, 2007).

Più di recente ancora Yin (2012) ha ribadito questi concetti evidenziando il vantaggio del multiple case approach e della sottesa replicazione che costituisce un ingrediente necessario per qualunque tentativo di sviluppo di una teoria robusta. 
Si è impiegata l'intervista come metodo di rilevazione potendo accedere, attraverso di essa, alle percezioni ed alle opinioni degli individui ed analizzare nel contempo i modi con cui gli attori sociali esaminati costruiscono la realtà che li circonda (Sala, 2010).

Il campionamento adottato è di tipo non probabilistico: le unità di analisi sono state scelte in relazione al fatto che le stesse avessero sperimentato in prima persona il fenomeno analizzato e ne avessero avuto una adeguata conoscenza (Rubin e Rubin, 2005).

Le imprese sono state individuate attraverso il sistema delle rivendite di materiali da costruzioni presenti in Puglia le quali, operando in costante contatto con le imprese edili del comparto, sono in possesso di un patrimonio informativo relativo agli attori in questione molto utile ai fini della ricerca.

È stato così effettuato un preliminare sondaggio telefonico per le province di Lecce, Brindisi, Taranto e Bari con i titolari delle stesse rivendite, finalizzato a conoscere i nominativi delle imprese di ogni area in possesso di entrambi i seguenti requisiti: 1) presenza di almeno cinque anni sul mercato; 2) realizzazione di almeno un intervento di costruzione di appartamenti a vendere nell'ultimo anno con un numero di unità abitative realizzate non inferiore a 12.

La lista così formata era inizialmente composta da 45 imprese. Di queste ne sono state scartate quattro in quanto da successive verifiche effettuate presso le locali camere di commercio è risultato che l'anzianità di iscrizione al registro imprese era inferiore ai cinque anni. Delle restanti 41, 17 non hanno dato la disponibilità a farsi intervistare, mentre 9 sono state eliminate dal campione dopo l'intervista in quanto le risposte fornite non sono risultate significative ai fini dello studio, non disponendo le persone intervistate (delegate dei titolari aziendali) delle necessarie informazioni loro richieste.

Nei restanti 15 casi, a rispondere alle domande sono stati i titolari delle imprese di costruzioni o i responsabili delle funzioni commerciali e tecniche, soggetti che stanti le piccole dimensioni aziendali del campione, concentrano funzioni e poteri decisionali molto ampi ed hanno dunque un contatto diretto ed immediato con la realtà oggetto di studio, essendo coloro in capo ai quali ricade il compito di adottare decisioni inerenti la struttura relazionale con le agenzie.

La raccolta dei dati è avvenuta dopo aver predisposto una traccia di intervista recante la lista di aree tematiche poi coperte nel corso del colloquio ed eseguendo la registrazione e successiva trascrizione dei contenuti delle interviste così da ottenere e conservare la fedele riproduzione delle informazioni raccolte e per rispettare le indicazioni provenienti dalla letteratura in materia (Silverman, 2000; Spradley, 1979; Kvale, 1996).

Le imprese del campione appartengono al comparto identificato dal codice Ateco 41.20.0, Costruzione di edifici di edilizia residenziale e non residenziale; l'ambito di operatività coincide quindi con la costruzione, e successiva vendita, di unità immobiliari destinate ad uso abitativo.

Hanno tutte un mercato di dimensione provinciale, 9 di esse sono della provincia di Lecce, 2 di quella di Taranto ed una di quella di Bari. 
Non è stato possibile quantificare le variabili economiche (fatturato, reddito, produzione ecc.) a causa di una diffusa "resistenza" a fornire dati quantitativi che in ogni caso sarebbe comunque difficile acquisire, trattandosi, almeno per una parte, di società di persone non tenute al deposito del bilancio presso la Camera di Commercio.

Sono caratterizzate da una forte impronta personalistica del titolare, o dei soci, che partecipano personalmente alla progettazione ed alla realizzazione dell'opera. Tutte le imprese hanno meno di 20 addetti.

\section{I fattori che determinano l'insourcing della funzione commerciale nelle imprese di costruzioni}

Le domande rivolte agli imprenditori hanno cercato di far luce in primo luogo sulla scelta adottata relativamente alla gestione della relazione con il cliente finale.

Le questioni affrontate hanno quindi indirizzato l'intervistato verso la trattazione delle variabili da lui ritenute rilevanti ed esplicative della scelta adottata ed a discutere delle motivazioni e della prospettiva dalla quale il costruttore osserva il suo rapporto sia con l'agente sia con il cliente.

In particolare, le interviste si sono focalizzate sui seguenti aspetti:

a) in or outsourcing?;

b) la complessità del prodotto ed il suo impatto sulla scelta distributiva;

c) le variabili competitive;

d) il rischio di opportunismo dell'agente;

e) la funzione del brand quale veicolo della scelta d'acquisto del prodotto.

Circa il primo aspetto, i risultati raccolti mostrano una netta propensione verso il canale diretto: le imprese, in 13 casi su 15, dichiarano di preferire lo svolgimento in proprio delle attività commerciali, con l'eventuale e complementare intervento dell'agenzia immobiliare, ma senza alcun mandato in esclusiva, impiegando risorse umane dedicate, non di rado coincidenti con la figura dello stesso titolare dell'impresa. La ragione di questo orientamento può ritrovarsi in una delle risposte, trascritta di seguito, che ben sintetizza il pensiero dei costruttori:

"Gli agenti immobiliari sanno poco di come sono fatti gli impianti, di come è composto l'involucro...in quanto... non si documentano, non hanno l'umiltà di apprendere, di chiedere, di fare delle verifiche in cantiere per capire come è strutturato a grandi linee e quali siano le basi del costruito".

Questa è dunque una delle ragioni che spingerebbe le imprese edili a non affidarsi al sistema delle agenzie ma a trattare direttamente, anche per via della seconda variabile indagata, vale a dire la complessità del prodotto abitativo. Su questo punto, dalle interviste, emerge una sostanziale condivisione del principio per cui sia necessario trattare tale prodotto in maniera completamente diversa da quanto avviene in altri mercati. L'impegno economico legato all'acquisto, le motivazioni sottostanti, la necessità di approfondite informazioni, anche di natura tecnica, sono 
tutti elementi che richiedono una specifica attenzione nelle varie fasi della vendita. Su questo si nota una diffusa diffidenza, ed anche una sentita critica verso gli agenti, che commetterebbero un errore di fondo, quello di trattare il prodotto abitativo come una commodity, allestendo le proprie vetrine come quelle "dei supermercati", trattando la casa "alla stregua di un normale acquisto commerciale, e non di un progetto di vita come invece è".

Vi è dunque una percezione negativa dell'effettivo apporto garantito dall'agente, il quale non assicurerebbe la disponibilità di informazioni complete per il cliente, e non sarebbe in grado di farlo sentire a suo agio durante la trattativa e nelle fasi preliminari.

Viene fatto notare che nella maggior parte dei casi egli non fa altro che accompagnare il cliente presso il costruttore perché quest'ultimo dia tutte le informazioni inerenti l'immobile, di fatto quindi il suo contributo si limiterebbe alla presentazione di un nuovo cliente e poco più.

"Le agenzie con le quali abbiamo avuto rapporti in passato ci hanno dato l'impressione di non essere professionali, anche nella descrizione dell'appartamento.

Quasi sempre l'agente ha portato il cliente da noi, quindi tutte le informazioni inerenti l'immobile siamo stati sempre noi a fornirle all'acquirente.

Ci siamo quindi resi conto che la collaborazione dell'agente era minima, non ci toglieva del lavoro. L'unica attività svolta è stata di individuazione e segnalazione del cliente".

Questo aspetto viene ribadito da gran parte degli intervistati che hanno anche giustificato questa tendenza degli acquirenti a parlare direttamente con loro sia per il convincimento di poter risparmiare sul prezzo (in particolare il costo della provvigione), sia per la possibilità di prospettare più adeguatamente le necessità legate al fabbricato in costruzione ed alle modalità di pagamento.

La personalizzazione sembra essere una tipicità dei rapporti con il cliente finale seppure, occorre precisarlo, per via della piccola dimensione aziendale del campione che, in più casi, ha evidenziato che il modello relazionale non potrebbe funzionare se la struttura aziendale o l'intervento edilizio fossero più grandi.

L'altra variabile esplicativa analizzata nelle scelte dei costruttori riguarda le politiche competitive e di prezzo. Si è analizzato quale sia l'impatto prodotto sul piano competitivo dalla scelta in/outsourcing. Le risposte si sono soffermate in particolare sul controllo dei costi e quindi sul presidio del cantiere e delle fasi esecutive durante tutta la durata delle operazioni, compresa quella commerciale. Questo monitoraggio costante e sistematico è considerato essenziale per contenere i costi di produzione e per essere competitivi sul prezzo e sulla qualità dell'opera.

"Parlando anche con altri colleghi ci rendiamo conto che delegano molto e questo impedisce loro di monitorare la qualità del costruito, il tecnico delegato non sempre è esperto e comunque non ha lo stesso interesse che ha il costruttore.

Noi peraltro la costruzione non la eseguiamo direttamente e questo ci dà più tranquillità nel controllo, superiamo cosi molti problemi di gestione del cantiere che scarichiamo sull'esecutore. Per cui possiamo controllare meglio ed in modo qualitativamente migliore le fasi della costruzione. 
Se un nostro operario commette un errore siamo noi a pagarne le conseguenze, se è il cottimista che sbaglia il contratto stabilisce le conseguenze ed i costi relativi".

"Il nostro punto di forza nei confronti dei concorrenti è sicuramente la disponibilità mostrata verso il cliente, sia per i pagamenti che per gli interventi e le modifiche.

Abbiamo sempre mostrato ampia flessibilità, diciamo che ci distinguiamo proprio per la nostra capacità di ascolto.

Ciò è possibile proprio perché gestiamo direttamente la vendita e perché trasformiamo la provvigione dell'agente in sconto per il cliente; ciò é possibile per cantieri vicini alla sede, dove siamo conosciuti, non abbiamo bisogno di intermediari, per cantieri più lontani invece, non avendo una conoscenza del mercato di riferimento, ci appoggiamo ad agenzie".

Per quanto riguarda i possibili comportamenti opportunistici da parte dell'agente, diverse sono state le prese di posizione, anche abbastanza "vibrate", assunte dagli intervistati.

Il primo esempio è dato da un costruttore che addebita agli agenti la responsabilità di creare, a proprio vantaggio, una cattiva immagine del costruttore $\mathrm{e}$ della sua onestà ed etica professionale. In particolare viene fatto notare come:

Nell'immaginario collettivo il costruttore è colui che vuole approfittare del cliente, ed in questo esistono precise responsabilità da parte delle agenzie immobiliari. Se $i$ costruttori, insieme ai progettisti che sono il volano di tutto il processo, fossero più consapevoli dell'importanza del proprio ruolo e facessero "massa critica", non assisteremmo a fenomeni come quello che porta a far fare il prezzo agli agenti.

Non esiste nulla di più sbagliato del "prezzo a mq", eppure è quello che succede. Non si può "omogeneizzare" tutto come oggi avviene a causa delle agenzie.

L'opportunismo verrebbe alimentato da un problema normativo che determina

"una discrasia tra la responsabilità del costruttore e quella dell'agente, da cui deriva un forte sbilanciamento in favore di quest'ultimo; il costruttore infatti ha una responsabilità decennale su ciò che costruisce, l'agente non ne ha nessuna".

Lo sbilanciamento è troppo netto e richiede un'attenta gestione dell'impresa, anche nelle fasi commerciali, per le quali si impone un contatto diretto.

Infine la scelta di insourcing è legata alla notorietà dell'impresa di costruzioni sul mercato di riferimento e quindi alla forza del suo brand. In tal senso sono emerse risposte sostanzialmente univoche che ne riconoscono l'importanza quale asset fondato sulla notorietà del nome sul mercato locale e sul patrimonio fiduciario costruito nel corso degli anni.

"La mia azienda è molto conosciuta sul mercato locale grazie alla capacità che le generazioni precedenti alla mia hanno dimostrato, legando il nostro nome alla qualità del costruito, i nostri clienti quindi ci scelgono per questo".

Il cliente si rivolge all'impresa perché conosciuta sul mercato locale grazie a precedenti esperienze di conoscenti ed amici, che attivano un positivo passaparola 
che rende irrilevante il ricorso ai tradizionali strumenti di comunicazione come la pubblicità:

"Non abbiamo mai fatto molta pubblicità, o meglio l'abbiamo fatta ma in modo sporadico. Il fatto di pubblicizzarsi non è molto importante nel caso di una piccola azienda come la nostra, che essendo una realtà giovane si differenzia attraverso il passaparola che cementifica il rapporto tra con in nostri clienti".

"Più che il marketing, ad attrarre il cliente è la conoscenza diretta delle persone. Chi viene da noi lo fa per una questione di fiducia, affidabilità, conoscenza diretta. Il contatto con il cliente è essenziale".

Oltre al passaparola ed alla notorietà della tradizione aziendale, il cliente apprezza anche "la continua, costante e meticolosa cura" dei lavori, il fatto che $\mathrm{i}$ titolari siano direttamente presenti in cantiere dove arrivano sin dalle prime ore della giornata.

L'assistenza costituisce uno dei più significativi punti di forza della struttura aziendale, rafforzativi dello stesso brand; di frequente è lo stesso titolare dell'azienda che gestisce direttamente il rapporto, dalla firma del preliminare, alla scelta della distribuzione degli ambienti, in ogni passo, e questo è ricercato ed apprezzato dall'acquirente.

\section{I fattori che spingono per l'outsourcing o per una soluzione "mista"}

Delle quindici imprese intervistate, solo due hanno dichiarato di aver esternalizzato le attività di vendita. Particolarmente interessanti appaiono le risposte di una delle due, che ha esposto in modo chiaro e netto la propria preferenza per l'outsourcing. Si tratta di un'impresa che lavora, sin dal momento della sua costituzione (avvenuta 30 anni fa) sempre con la stessa agenzia: il caso merita di essere richiamato per la sua particolarità e per l'approccio adottato ancora oggi; l'amministratore sottolinea che:

"l'agenzia deve fare il consulente dell'impresa, in quanto quest'ultima non può avere la presunzione di "comandare" il mercato. Il nostro rapporto si è proprio sviluppato su queste basi: è l'agenzia che ci segue e ci indica ciò che il mercato vuole. È quanto è avvenuto ad esempio verso la metà degli anni '80 quando ci fu suggerito di comprare dei suoli in una zona assolutamente "improbabile" con l'indicazione della tipologia di immobili e delle modalità di finanziamento da adottare. Nonostante le iniziali titubanze seguimmo comunque il loro suggerimento, andò benissimo! Riuscimmo a vendere $i 32$ appartamenti ed anche $i$ successivi che sulla base di questo "successo" decidemmo di costruire.

Fu l'inizio di una serie di operazioni tutte ben riuscite che si sono innestate su questo modello cooperativo e sinergico. La stessa agenzia ci suggerì nel seguito tipologie immobiliari differenti sulla base delle sopraggiunte esigenze abitative che nella stessa area di mercato stavano emergendo, e queste proposte furono da noi seguite e coronate da successo". 
Il secondo caso riguarda una piccola impresa che per le attività di vendita si avvale della collaborazione di due agenzie alle quali vengono assegnati ambiti di mercato differenti e questo perché

"il costruttore non ha il tempo di fare quello che fanno le agenzie che chiamano il cliente, lo assistono, lo informano",

attività per le quali l'impresa non è attrezzata o alle quali non intende dedicare del tempo che viene destinato ad altre attività ritenute più importanti.

Un approccio differente è quello di chi si dichiara disposto a modificare la propria politica distributiva, passando da un canale diretto ad uno misto, per due sostanziali ragioni:

a) sfruttare la maggiore rigidità dell'agenzia relativamente al prezzo di vendita;

b) compensare la riduzione del livello delle vendite causato dalla crisi economica.

Per quanto riguarda il primo punto, viene osservato che

"l'agenzia nel fissare il prezzo al cliente non lo cambia perché avrebbe indicazioni di questo tipo da parte nostra...sarebbe più rigida nel mantenere le condizioni contrattuali rispetto a quanto non lo saremmo noi, che siamo più flessibili nel rapporto con il cliente che viene a comprare direttamente".

Sul secondo punto invece si è giustificato il cambiamento affermando che:

"Oggi è indispensabile rivolgersi all'agenzia immobiliare perché i tempi sono cambiati, noi per esempio abbiamo preventivamente fornito il progetto all'agenzia al fine di capirne la fattibilità e la vendibilità degli appartamenti, nonché le esigenze del mercato.

Ed anche per far piazzare almeno il 20 per cento prima di iniziare".

"Attualmente adottiamo un canale misto, ci avvaliamo in esclusiva di un'agenzia di famiglia e nello stesso tempo facciamo direttamente. La motivazione di questa scelta è legata al ciclo economico favorevole che abbiamo avuto sino ad un paio di anni fa, in questa fase di stallo del mercato stiamo valutando la possibilità di esternalizzare completamente questo servizio poiché riteniamo sia più opportuno che tutta la contrattualistica ed il contatto con il cliente vengano seguite da professionisti esterni specializzati.

$C$ 'è anche un'altra ragione: un agente riuscirebbe a dire "no" molto meglio di quanto non saremmo in grado di farlo noi, pensiamo cioè che se un cliente rivolgendosi direttamente a noi ci chiedesse di fare delle modifiche al fabbricato includendole nel prezzo, noi saremmo più propensi ad accontentarlo, con un aggravio di costi che l'agenzia, in una situazione simile, ci eviterebbe non essendo autorizzata a modificare il prezzo fissato a monte.

Infine abbiamo necessità di capire cosa vuole il mercato e l'agenzia ce lo può dire (o dovrebbe sapercelo dire)".

\section{Conclusioni, limiti della ricerca ed implicazioni manageriali}

La ricerca ha evidenziato un prevalente orientamento ad internalizzare la funzione commerciale e di vendita nel convincimento, espresso dalla quasi totalità, 
che le agenzie immobiliari non possiedano le necessarie competenze e professionalità che la vendita di un prodotto complesso, quale è quello abitativo, richiede. Inoltre, secondo i costruttori, vi è una netta preferenza del cliente per un dialogo diretto con loro, per i vantaggi che questa interazione consente in termini di modifiche sulla costruzione (in corso d'opera o a lavori completati), di riduzioni di prezzo (in particolare per quanto riguarda la provvigione), di completezza ed attendibilità delle informazioni.

La scelta di in/outsourcing è peraltro legata alla notorietà ed alla awareness del brand del costruttore, che pur non essendo il frutto di una specifica politica di marketing e di investimenti pubblicitari e di comunicazione ad hoc, risente comunque positivamente ed in maniera consistente dell'affidabilità delle realizzazioni edili e della serietà nei comportamenti che le imprese sono riuscite a dimostrare nel corso del tempo.

La tabella seguente mostra una sintesi delle variabili esplicative delle scelte di in/outsourcing, così come emerse dalle interviste.

\section{Tab. 1: Sintesi delle principali motivazioni all'insourcing} ed all'outsourcing emerse dalla ricerca

\begin{tabular}{|c|c|}
\hline INSOURCING & OUTSOURCING \\
\hline $\begin{array}{l}\text { Necessità di fornire al cliente informazioni } \\
\text { complete ed adeguate sul costruito ("gli agenti } \\
\text { sanno poco di come è composto l'involucro...") }\end{array}$ & $\begin{array}{l}\text { Possibilità di sfruttare la rigidità delle agenzie } \\
\text { nell'applicazione del prezzo di vendita } \\
\text { (“...l'agenzia riesce ad essere più rigida di noi } \\
\text { nel mantenere il prezzo di vendita...) }\end{array}$ \\
\hline $\begin{array}{l}\text { Banalizzazione del prodotto da parte degli agenti } \\
\text { (“...le vetrine sembrano quelle di un } \\
\text { supermercato...") }\end{array}$ & $\begin{array}{l}\text { Specializzazione dell'agenzia nelle attività } \\
\text { contrattualistiche ed amministrative (“...è più } \\
\text { opportuno che queste attività vengano seguite } \\
\text { da professionisti specializzati...") }\end{array}$ \\
\hline $\begin{array}{l}\text { Propensione del cliente a relazionarsi } \\
\text { direttamente con il costruttore ("vogliono parlare } \\
\text { di persona con me...") }\end{array}$ & $\begin{array}{l}\text { Maggiore conoscenza del mercato da parte } \\
\text { dell'agente ("... abbiamo necessità di capire cosa } \\
\text { vuole il mercato e l'agenzia ce lo può dire....") }\end{array}$ \\
\hline $\begin{array}{l}\text { Possibilità di applicare prezzi più competitivi } \\
\text { ("trasformiamo la provvigione dell'agente in } \\
\text { sconto per il cliente") }\end{array}$ & $\begin{array}{l}\text { Necessità di contare su un partner per le attività } \\
\text { commerciali ("...l'agenzia deve fare il consulente } \\
\text { dell'impresa") }\end{array}$ \\
\hline $\begin{array}{l}\text { Superiorità del brand del costruttore ("Leghiamo } \\
\text { il nostro nome alla qualità del costruito, i nostri } \\
\text { clienti ci scelgono per questo") }\end{array}$ & $\begin{array}{l}\text { Riduzione dei costi di modifiche in corso d'opera } \\
\text { ("...l'agenzia non sarebbe autorizzata a } \\
\text { modificare il progetto") }\end{array}$ \\
\hline $\begin{array}{l}\text { Assistenza diretta al cliente (“...fa la differenza } \\
\text {..”) }\end{array}$ & $\begin{array}{l}\text { Concentrazione sul core business (“...non } \\
\text { abbiamo il tempo di fare quello che fanno le } \\
\text { agenzie...") }\end{array}$ \\
\hline $\begin{array}{l}\text { Scarsa propensione al rischio delle agenzie } \\
\text { (“...le agenzie si tirano spesso indietro senza } \\
\text { assumersi responsabilità concrete”) }\end{array}$ & \\
\hline
\end{tabular}

Fonte: Elaborazione dell'autore

Nell'attuale situazione di crisi però le imprese percepiscono l'esigenza di aprirsi alla collaborazione con il sistema delle agenzie immobiliari per ottenere un supporto nell'intercettare nuova clientela. Tuttavia l'idea è, comunque, mantenere all'interno la gestione della relazione, nelle differenti fasi del processo d'acquisto. 
Un aspetto preponderante nel campione che, è opportuno precisarlo, è totalmente composto da imprese di piccola dimensione, con un raggio di operatività ed un ambito di mercato strettamente provinciale.

Proprio la dimensione aziendale e del mercato servito sembrano essere alla base degli orientamenti emersi dalla ricerca i cui risultati presentano dunque il limite di non poter essere generalizzati a tutto l'universo delle imprese edili, in particolare di quello costituito da imprese medio-grandi, per le quali appare necessaria una estensione dello studio così da poter verificare le tendenze in esse prevalenti.

Si ritiene inoltre che possibili approfondimenti futuri debbano interessare anche l'analisi delle performance conseguibili in relazione alla scelta distributiva. Un aspetto non trattato nel presente lavoro ma che ne costituirà il necessario corollario da integrare attraverso l'introduzione di specifici indicatori.

Il lavoro qui presentato offre degli spunti di riflessioni agli operatori della filiera delle costruzioni, ed in particolare alle agenzie immobiliari, poiché dà contezza degli orientamenti e degli atteggiamenti dei costruttori nei confronti del loro operato. Dalle considerazioni espresse nel corso delle interviste appare chiaro come le imprese edili, in buona parte dei casi, ritengano l'agente un soggetto poco professionale e scarsamente competente in merito agli aspetti tecnici del costruito, che adotta un approccio inadeguato alle specificità del prodotto, che non partecipa alle attività di cantiere e che quindi non é in grado di dare risposte esaurienti alle richieste del cliente.

A questa posizione, che abbiamo visto essere prevalente nel campione, si contrappone quello di una minoranza che, al contrario, considera l'agenzia un partner insostituibile per la sua specializzazione in particolari attività (propedeutiche alla vendita), quali quelle amministrative e legali, oltre che per l'approfondita conoscenza del mercato e delle sue dinamiche.

Tali risultati impongono una riflessione ad entrambi gli attori dell'industria edile, poiché evidenziano una diffusa diffidenza dei costruttori che di fatto impoverisce il potenziale che il rapporto potrebbe esprimere se, al contrario, venisse costruito su solide basi fiduciarie e di reciproca collaborazione. L'evidente complementarietà dei ruoli infatti è di per sé una circostanza che dovrebbe condurre entrambi a ricercarsi, ed a riservarsi, degli spazi di reciproca esclusiva che ottimizzando l'uso delle risorse umane e professionali presenti in entrambi gli ambiti, offrano al cliente un servizio più soddisfacente $\mathrm{e}$ completo $\mathrm{e}$ generino un miglioramento delle proprie performance.

Riflettere sui risultati della ricerca può quindi costituire un utile esercizio per provare un dialogo costruttivo e collaborativo che nell'attuale fase di mercato, in cui peraltro gli stessi costruttori stanno comunque gradualmente modificando il proprio atteggiamento, rappresenta un percorso assolutamente necessario.

\section{Bibliografia}

ABIS M. (2009), "La casa degli italiani in tempi di crisi", Micro e Macro Marketing, n. 2, pp. 303-307. 
ALBINO V., COSTANTINO N., SIVO G. (2000), Le costruzioni: mercato e impresa, Carocci, Roma.

ANTHONY R.N., GOVINDARAJAN V., MACRI' D.D. (2006), Management control system. Tecniche e processi per implementare le strategie, McGraw-Hill, Milano.

ANTONIDES G., VAN RAAIJ W.F. (1988), Consumer Behavior: a European perspective, John Wiley \& Sons, New York, NY.

BADEN-FULLER C., TARGETT D., HUNT B. (2000), "Outsourcing to Outmanoeuvre: Outsourcing redefines competitive strategy and structure", European Management Journal, vol. 18, n. 3, pp. 285-295.

BAGOZZI R.P. (1974), "Marketing as an organized behavioral system of exchange", Journal of Marketing, n. 38, pp. 77-81.

BAGOZZI R.P. (1975), “Marketing as Exchange”, Journal of Marketing, n. 39, pp. 32-39.

BALDINI M. (2010), La casa degli italiani, Il Mulino, Bologna.

BITNER M.J. (1990), "Evaluating service encounters: the effects of physical surroundings and employee responses", Journal of Marketing, vol. 54, pp. 69-82.

BOYD D., CHINYIO E (2006), Understanding the construction client, Blackwell Publishing, Oxford.

BRASSINGTON F., PETTITT S. (2000), Principles of Marketing. $2^{\text {nd }}$ ed., Financial Times Prentice-Hall, Upple Saddle River, NY.

BROWN J.J., REINGEN P.H. (1987), "Social ties and word-of-mouth referral behavior", Journal of Consumer Research, vol. 14, December, pp. 350-362

CASTALDO S. (1994), Le relazioni distributive. La dimensione collaborativa nei rapporti industria-distribuzione, Egea, Milano.

CASTALDO S. (2005), Fiducia e relazioni di mercato, Il Mulino, Bologna.

CASTALDO S., SABBADIN E. (2009), Innovazione e collaborazione nella filiera delle costruzioni, Edizioni Tecniche Nuove.

COASE R.H. (1937), "The nature of the firm", Economica, vol. 4, n. 16, pp. 386-405.

DISHMAN P. (1996), "Exploring strategies for companies that use manifacturer's representatives as their sales force", Industrial Marketing Management, vol. 25, n. 4, pp. 453-461.

DUBOIS A., GIBBERT M. (2010), "From complexity to transparency: managing the interplay between theory, method and empirical phenomena in IMM case studies", Industrial Marketing Management, vol. 39, pp. 129-136.

DUBOIS A., GADDE L.E. (2013), "Systematic combining. A decade later", Journal of Business Research, http://dx.doi.org/10.1016/j.jbusres.2013.03.036.

DWYER R., SCHURR P., OH S. (1987), “Developing Buer-Seller Relationshpis”, Journal of Marketing, vol. 51, n. 2, pp. 11-27.

EASTON G. (2010), "Case study research. A critical realistic approach", Industrial Marketing Management, vol. 39, n. 1, pp. XX.

EISENHARDT K.M. (1989), "Building theories from case study research", Academy of Management Review, vol. 14, n. 4, pp. 532-550.

EISENHARDT K.M. (1991), "Better stories and better constructs: The case for rigor and comparative logic", Academy of Management Review, vol. 16, n. 3, pp. 620-627.

EISENHARDT K.M., GRAEBNER M.E., (2007), "Theory building from cases: Opportunities and Challenges", Academy of Management Review, vol. 50, n. 1, pp. 25-32.

ELMUTI D., KATHAWALA Y., MONIPPALLIL M. (1998), "Outsourcing to Gain a Competitive Advantage", Industrial Management, May/Jun; vol. 40, n. 3, pp. 20-24. 
ENGEL J.F., BLACKWELL R.D., MINIARD P.W. (1995), Consumer behavior, $8^{\text {th }}$ ed., The Dryden Press, Hinsdale, IL.

FAIT M. (2012), "Tipico e turismo: un network per creare valore. Il caso del Franciacorta", Sinergie n. 89, pp. 255-274.

FINIZIO M. (2011), "La casa cablata si vende in fretta", Casa 24 Plus dell'1 dicembre 2011.

FLOREDDU P.B., CABIDDU F. (2013), "Innovating distribution channels for competitive advantage", XXV Convegno Annuale di Sinergie, "L'innovazione per la competitività delle imprese", Ancona, 24-25 ottobre 2013.

FREYTAG P.V., CLARKE A.H., EVALD M. (2012), "Reconsidering outsourcing solutions", European Management Journal, vol. 30, pp. 99-110.

GAMESON R.N. (1991), Clients and professionals: the interface. Practice Management: New perspective for the construction professional, Spon, London.

GANDOLFO A., SBRANA R. (2007), Contemporary Retailing. Il governo dell'impresa commerciale moderna. Giappichelli, Torino.

GENCO P. (2006), "La dematerializzazione del territorio e dell'impresa: l'impresa progetto", Sinergie, n. 70, pp. 99-115.

GEYSKENS I., STEENKAMP J., SCHEER L.K., KUMAR N. (1996), “The effects of trust and interdependence on relationship commitment: a trans-Atlantic study. International Journal of Research in Marketing, n. 13, pp. 303-317.

GRANDORI A.(1999), Organizzazione e comportamento economico, Il Mulino, Bologna.

GREGORI G.L. (2001), L'evoluzione del processo di outsourcing nelle strategie di sviluppo delle imprese, Giappichelli, Torino.

GREWAL R., COMER J.M., MEHTA R. (1999), "Does trust determine satisfaction in marketing channel relationships? The moderating role of exchange partner's price competitiveness", Journal of Business to Business Marketing, vol. 6, n. 1, pp. 1-18.

GOLINELLI G.M. (2004), "Ridefinire il valore della marca", Sinergie, n. 63, pp. 221-227.

GUIDO G. (2000), Economia e gestione delle imprese. Principi, schemi, modelli, FrancoAngeli, Milano.

GULATI R., BRISTOW D. (2005), “The impact of relationships-specific adaptations and information exchange on sales agents' role salience", The Marketing Management Journal, vol. 15, n. 1, pp. 126-139.

GUMMESSON E. (2000), Qualitative methods in management research, Thousand Oaks, Sage Publications, Inc.

HINTERHUBER H., STUHEC U. (1996), "Competenze Distintive e Outsourcing Strategico", Finanza, Marketing e Produzione, vol. 14, n. 4, pp. 43-82.

KABADAYI S. (2011), "Choosing the right multiple channel system to minimize transiction costs", Industrial Marketing Management, vol. 5, n. 40, pp. 763-773.

KALAIGNANAM K., VARADARAJAN R. (2012), "Offshore outsourcing of customer relationship management: conceptual model and propositions", Journal of the Academy Marketing Science, vol. 40, n. 12, pp. 347-363.

KIESSLING T., HARVEY M. (2004), "Global marketing networks and the development of trust: a dyamic capabilities perspective", Journal of Marketing Channels, vol. 11, n. 4, pp. 21-41.

KOTABE M., MOL M.J., MURRAY J.Y., PARENTE R. (2012), "Outsourcing and its implications for market success: negative curvilinearity, firm resources, and competition. Journal of the Academy Marketing Science, vol. 40, n. 2, pp. 329-346.

KVALE S. (1996), Interviews: an introduction to qualitative research interviewing, Sage, Thousand Oaks. 
JACKSON D.W., WIDMIER S., GIACOBBE R., KEITH J. (1999), "Examining the use of team selling by manufacturers' representatives: A situational approach", Industrial Marketing Management, vol. 28, n. 2, pp. 155-164.

JAP S.D., ANDERSON E. (2003), "Safeguarding interorganizational performance and continuity Under ex post opportunism", Management Science, vol. 49, n. 12, pp. 1684-1701.

JOHNSTON W.L., LEACH M.P., LIU A.H. (1999), "Theory testing using case studies in business-to-business research", Industrial Marketing Management, vol. 28, n. 3, pp. 201-213.

LAMBIN J.J. (2008), Marketing-driven management. Marketing strategico ed operative. McGraw-Hill, Milano.

LE BON J., HUGHES D.E. (2009), "The dilemma of outsourced customer service and care: research propositions from a transaction cost perspective", Industrial Marketing Management, vol. 38, n. 4, pp. 404-410.

LEVITT T. (1980), "Marketing Success through Differentiation of Anything", Harvard Business Review, vol. 58, n. 1, pp. 89-91.

LI J.J. (2012), "The alignment between organizational control mechanisms and outsourcing strategies: A commentary essay", Journal of Business Research, vol. 65, pp. 13841386.

LOE E. (2000), The value of architecture: context and current thinking, Royal Institute of British Architects Future Studies, London.

LONGO M.A. (2006), "Relazioni distributive, dinamiche competitive e teoria dei giochi", Sinergie, n. 70, pp. 249-274.

LUGLI G. (1976), Economia della distribuzione commerciale, Giuffrè, Milano.

MAIZZA A., IAZZI A., TRIO O., GRAVILI S. (2012), "La fiducia nelle relazioni BancaImpresa: quali conseguenze sullo sviluppo territoriale?", XXIV Convegno Annuale di Sinergie "Il territorio come giacimento di vitalità per l'impresa", Lecce, 18-19 ottobre 2012.

MALHOTRA N.K. (1993), Marketing Research: an applied orientation, Englewood Cliffs, N.J., Prentice Hall.

MASSARONI E., RICOTTA F., (2009), "Dal sistema impresa ai sistemi di imprese. Suggestini e limiti delle reti d'impresa", Sinergie, n. 80, pp. 3-29.

McQUISTON D. (2001), "A conceptual model for building and maintaining relationships between manufacturers' representatives and their principals", Industrial Marketing Management, vol. ????, n. 30, 165-181.

MILGROM P., ROBERTS J. (1994), Economia, Organizzazione e Management, Il Mulino, Bologna.

MORELLO M. (2011), "Soggiorno in movimento", Casa 24 Plus del 27 ottobre 2011.

MORGAN R.M., HUNT S.D. (1994), "The commitment-trust theory of relationship marketing", Journal of Marketing, vol. 58, n. 3, pp. 20-38.

MULLIN R., (1996), "Outsourcing: Managing th Outsourced Enterprise”, Journal of Business Strategy, vol. 17 , n. 4 , pp. $28-36$

NEVINS J.L., MONEY R.B. (2008), "Performance implication of distributor effectiveness, trust, and culture in import channels of distribution", Industrial Marketing Management, vol. 37, n. 1, pp. 46-58.

NORSA A. (a cura di) (2006), La gestione del costruire tra progetto processo e contratto, Franco Angeli, Milano.

PASTORE A. (1996), "I nuovi rapporti tra industria e distribuzione. Le aree e gli strumenti per la partnership”, Cedam, Padova. 
PASTORE P., RICCIARDI A. (2010), Outsourcing strategico. Tecniche di gestione, criticità, vantaggi competitivi, Franco Angeli, Milano.

PELLICELLI M. (2009), L'outsourcing e l'offshoring nell'economia dell'impresa, Giappichelli, Torino.

PIEKKARI R., PLAKOYIANNAKI E., WELCH C. (2010), "Good case research in industrial marketing: insights from research practice", Industrial Marketing Management, vol. 39, n. 1, pp. 109-117.

POLITECNICO DI MILANO (2009), L'Ict nel Real Estate: un potenziale innovativo non ancora sfruttato", documento prelevato dal sito www.osservatori.net.

PRAHALAD C.K., HAMEL G. (1990), "The core competence of corporation", Harvard Business Review, vol. 68, n. 3, pp. 79-91.

QUINN J.B., HILMER F.G. (1994), “Strategic outsourcing”, Sloan Management Review, vol. 35, n. 4, pp. 43-55

RAJAGOPAL (2008), "Outsourcing salespeople in building arousal towards retail buying", Database Marketing \& Customer Strategy Management, vol. 15, n. 2, pp. 106-118.

RAPP A. (2009), "Outsourcing the sales process: Hiring a mercenary sales forces", Industrial Marketing Management, vol. 38, pp. 411-418.

RICCIARDI A. (2001), L'outsourcing strategico. Modalità operative, tecniche di controllo ed effetti sugli equilibri di gestione, Franco Angeli, Milano.

ROSS W.T., DALSACE F., ANDERSON E. (2005), "Should you set up your own sales force or should you outsource it? Pitfalls in the standard analysis", Business Horizons, vol. 48, n. 4, pp. 23-26.

RUBIN H.J., RUBIN I.S. (2005), Qualitative interviewing: the art of hearing data, Sage, Thousand Oaks.

RULLANI E. (2010), "Impresa e produzione di valore nell'era della complessità”, Sinergie, n. 81 , pp. $225-242$.

SABBADIN E. (2007), Mercato e distribuzione nell'edilizia. La collaborazione tra industria e commercio in una filiera complessa, Edizioni Tecniche Nuove, Milano.

SALA E. (2010), L'intervista, in De Lillo A. (a cura di), Il mondo della ricerca qualitativa, Utet, Torino.

SILVERMAN D. (2000), Interpreting qualitative data, Sage, London.

SPRADLEY J.P. (1979), The ethnographic interview, Holt, Rinehard and Winston, New York.

SPRANZI A. (1972), "I rapport industria-distribuzione", in Guatri L. (a cura di), Manuale di Marketing, Isedi, Milano.

SULLIVAN J., PETERSON R.B. (1982), "Factors associated with trust in JapaneseAmerican joint-ventures", International Management Review, vol. 22, n. 2, pp. 30-40.

THELEN S., YOO B., MAGNINI V.P., (2011), "An examination of consumer sentiment toward offshored service", Journal of the Academy of Marketing Science, n. 39 (April), pp. 270-289.

TIDD J., BESSANT J., PAVITT K. (1999), Management dell'innovazione, Guerini e Associati, Milano.

TRIO O. (2008), "Le costruzioni, un'industria a banda larga di tecnologia", in Trio O. (a cura di), Innovazione e risparmio energetico, nuove sfide per il cambiamento dell'edilizia abitativa, Franco Angeli, Milano.

TRIO O. (2010a) L'industria edile. Attori, processi, mercati, Cacucci, Bari.

TRIO O. (2010b), "L'innovazione nell'edilizia abitativa tra risparmio energetico e nuovi materiali", L'industria, vol. 2, pp. 277-301. 
VACCÀ S. (1963) I rapporti industria-distribuzione nei mercati dei beni di consumo. Giuffrè, Milano.

VARALDO R. (1971) Potere e conflitti nei canali di distribuzione, Etas, Pisa.

VARALDO R., DALLI D. (1989), "Le relazioni strategiche tra industria e distribuzione", Sinergie, n. 19, pp. 13-48.

VARALDO R., DALLI D. (2003), "Le relazioni strategiche tra industria e distribuzione", Sinergie, n. 61-62, pp. 255-297.

VENTRICELLI G. (2004), Outsourcing. Conviene davvero esternalizzare?, Etas, Milano.

WAGNER W., KIRCHLER E., BRANDASATTER H. (1984) "Marital relationships and purchasing decisions - to buy or not to buy? That is the question", Journal of Economic Phsychology, vol. 5, n. 2, pp. 139-157.

WEINRAUCH D., STEVENS M., CARLSON R. (1997), "Converting from independent to employee sales forces: the role of perceived switching costs", Journal of Marketing Research, vol. 29, n. 2, pp. 101-115.

WILLIAMSON O. (1978), Markets and Hierarchies, The Free Press, New York.

YIN R.K. (1984), Case Study Research: Design and Methods, Sage Publications, California.

YIN R.K. (2012), "Applications of case study research", Sage Publications, Thousand Oaks. 